schen und elektrischen Eigenschaften von Cäsium Antimon-Schichten verschiedener Zusammensetzung gemessen. Im Anschluß daran wurden die gleichen Untersuchungen an Cäsium-Wismut-Schichten ausgeführt ${ }^{6}$. Für zwei charakteristische Formierzustände, denen man auf Grund des Formierdiagramms ${ }^{6}$ und des $\mathrm{Zu}$ standsdiagramms ${ }^{4}$ des Systems $\mathrm{Bi}-\mathrm{Cs}$ die Zusammensetzungen $\mathrm{CsBi}_{2}$ und $\mathrm{Cs}_{3} \mathrm{Bi}$ zuschreiben kann, wurden die in Tab. 1 angegebenen Werte für die optische Absorptionskante, die Grenzenergie des äußeren Photoeffektes, die thermische Austrittsarbeit und die Aktivierungsenergien der Leitfähigkeit gefunden.

\begin{tabular}{|l|c|c|}
\hline & $\begin{array}{c}\text { Formier- } \\
\text { zustand I } \\
\mathrm{CsBi}_{2}\end{array}$ & $\begin{array}{c}\text { Formier- } \\
\text { zustand II } \\
\mathrm{Cs}_{3} \mathrm{Bi}\end{array}$ \\
\hline$\Delta E_{\text {opt }}$ (Absorption) & $<0,55 \mathrm{eV}$ & $<0,55 \mathrm{eV}$ \\
$\Delta E_{\text {phot (äuß. Photoeff.) }}$ & $1,65 \mathrm{eV}$ & $1,5 \mathrm{eV}$ \\
$\Delta E_{\text {therm (therm. Emission) }}$ & $0,9 \mathrm{eV}$ & - \\
$\Delta E_{\sigma}$ (Leitfähigkeit) & $0,4 \mathrm{u.} 0,7 \mathrm{eV}$ & - \\
\hline
\end{tabular}

Tab. 1 .

Die Einzelheiten der Zuordnung dieser Energien zu den Niveauabständen des Bändermodells sind zwar noch nicht eindeutig; ein Zusammenhang jedoch tritt bereits deutlich hervor. Während bei $\mathrm{Cs}_{3} \mathrm{Sb}$ das Wahrscheinlichere der mit den Messungen verträglichen Bändermodelle einen Bandabstand von 1,6 eV besitzt ${ }^{5}$, hat das entsprechende Bändermodell für die analoge Verbindung $\mathrm{Cs}_{3} \mathrm{Bi}$ einen Bandabstand $<0,55 \mathrm{eV}^{6}$. Beim Übergang von $\mathrm{Sb} z u \mathrm{Bi}$, d. h. zu einem schwereren Ele-

\begin{tabular}{|c|c|c|c|c|c|c|}
\hline \multicolumn{2}{|c|}{$\mathrm{A}^{\mathrm{II}} \mathrm{B}^{\mathrm{IV}}$} & \multicolumn{2}{|c|}{$\mathrm{A}^{\mathrm{II}} \mathrm{B}^{\mathrm{VI}}$} & \multicolumn{2}{|c|}{$\mathrm{A}^{\mathrm{III}} \mathrm{B} \mathrm{V}$} & $\mathrm{A}^{\mathrm{I}} \mathrm{B} \mathrm{V}$ \\
\hline $\mathrm{Mg}_{2} \mathrm{Si}$ & 0,77 & $\mathrm{CdS}$ & 2,0 & AlP & 3 & $\mathrm{Cs}_{3} \mathrm{Sb} \quad 1,6$ \\
\hline $\mathrm{Mg}_{2} \mathrm{G}$ & 0,74 & $\mathrm{CdSe}$ & 1,8 & AlAs & 2,2 & $\mathrm{Cs}_{3} \mathrm{Bi}<0,55$ \\
\hline $\mathrm{Mg}_{2} \mathrm{Sr}$ & 0,36 & $\mathrm{CdTe}$ & 1,45 & $\mathrm{AlSb}$ & 1,65 & \\
\hline $\mathrm{Ca}_{2} \mathrm{Si}$ & 1,9 & $\mathrm{ZnS}$ & 3,7 & InP & 1,25 & \\
\hline $\mathrm{Ca}_{2} \mathrm{Sn}$ & 0,9 & $\mathrm{ZnSe}$ & 2,6 & InAs & 0,47 & \\
\hline $\mathrm{Ca}_{2} \mathrm{~Pb}$ & 0,46 & $\mathrm{ZnTe}$ & 2,2 & $\mathrm{InSb}$ & 0,27 & \\
\hline
\end{tabular}

Tab. 2. Bandabstände $\Delta E$ in $\mathrm{eV}$.

ment der gleichen Gruppe des periodischen Systems, beobachtet man also eine Verkleinerung des Bandabstandes.

Dies Verhalten stimmt mit der bei mehreren anderen intermetallischen Verbindungen beobachteten Tendenz überein (Tab. 2).

${ }^{6}$ H. G. Clerc u. G. Wallis, Ann. Phys., Lpz. (erscheint demnächst).

\section{Die Zündspannung des Anodenbogens}

\section{Von Wilhelm MerL}

Physikalisches Laboratorium der Firma Dr. E. Dürrwächter - DODUCO - KG., Pforzheim

(Z. Naturforschg. 11 a, 1041-1042 [1956] ; eingeg. am 31. Oktober 1956)

Entlädt man einen Kondensator durch Schließen eines Kontaktes, so setzt der Stromfluß vor der metallischen Berührung der Elektroden ein, selbst wenn die Kondensatorspannung weit unter dem Minimum der PAschenKurve liegt. Dieser in neuerer Zeit erforschte Durchschlagsmechanismus bei Elektrodenabständen unter $10^{-4} \mathrm{~cm}$ und reinen Metalloberflächen läßt sich nicht mit der TownsEnd-Vorstellung in Einklang bringen ${ }^{1,2}$. Aus den Untersuchungen von Germer und Mitarb. geht hervor, daß zur Zündung der kurzzeitigen Entladungen Feldstärken von rund $10^{7} \mathrm{~V} / \mathrm{cm}$ erforderlich sind ${ }^{3}$. Dieser Wert deutet, zusammen mit den aus Schmelzspuren an den Bogenfußpunkten ermittelten Stromdichten von $10^{6}$ bis $10^{8} \mathrm{~A} / \mathrm{cm}^{2}$, darauf hin, daß die Zündung und Erhaltung des Bogens durch Feldelektronenemission erfolgt. Der Anteil der Ionen am Gesamtstrom nimmt mit der Entladungsdauer bis zu $15 \%$ zu und wird durch Verdampfung der Anode geliefert 4, 5. Neutralisierte Ionen und ein Teil des Metalldampfes lagern sich dabei auf der Kathode in Form kleiner Kuppen ab, deren

1 W. S. Boyle u. P. Kisliuk, Phys. Rev. 97, 255 [1955].

2 L. Loes, Sparks in very short gaps, at high pressure and in vacuum, in S. F LüGGE , Handb. d. Phys. 22, 527 [1956].
Volumen bei Spannungen unter 70 Volt der Kondensatorenergie proportional ist. Diese Eigenschaft des Bogens wurde ausgenützt, um mit verhältnismäßig geringem experimentellem Aufwand das schwer erfaßbare Gebiet um die Bogenmindestspannung näher zu untersuchen. Bisher konnte der Anodenbogen aus meßtechnischen Gründen nur bis zu 30 Volt herab oszillographisch verfolgt werden.

Durch wiederholtes Entladen eines Kondensators über Edelmetall-Kontakte wurden die kathodenseitigen Ablagerungen auf mindestens $10^{-6} \mathrm{~cm}^{3}$ angehäuft und damit einer genauen Messung zugänglich gemacht. In einem Metallvolumen - Spannungs-Diagramm war dann die Zündspannung des Anodenbogens als ausgezeichneter Punkt zu erkennen. Die Versuchsanordnung bestand aus einem mit Au- bzw. Pd-Kontakten bestückten Resonanzschalter $(R=2 \mathrm{~m} \Omega, L \sim 0,06 \mu \mathrm{H})$, der einen $1-\mu \mathrm{F}$-Kondensator in der Sekunde 100-mal kurzschloß. Als Stromquellen dienten bis zu 12 Volt Blei- und Nickel-Kadmium-Sammler, darüber stromstarke Braunstein-Anodenbatterien, deren Spannung während der Versuchsdauer um weniger âls 0,05 Volt abnahm. Der Ladewiderstand des Kondensators von $2,2 \mathrm{k} \Omega$ genügte, um das Verhältnis der durch den Einschaltbogen beding. ten Stoffwanderung zu der beim Öffnen des Schaltkreises erzeugten ${ }^{6}$ größer als $10^{3}$ werden zu lassen, so daß sich eine Trennung von Ein- und Ausschaltvorgang erübrigte.

3 L. H. Germer u. W. S. Boyle, J. Appl. Phys. 27, 32 [1956].

4 P. Kisliuk, J. Appl. Phys. 25, 897 [1954].

5 W.S. Boyle u. L. H. Germer, J. Appl. Phys. 26, 571 [1955].

6 W. Merl, Elektrotechn. Z. A 77, 201 [1956]. 
In dem für die Kontakte verwendeten Feingold waren 0,05\% Verunreinigungen gelöst, in der Hauptsache Ag und $\mathrm{Cu}$ mit Spuren von $\mathrm{Fe}$ und $\mathrm{Ca}$. Die PalladiumKontakte enthielten noch rund 0,2\% Fremdmetalle, und zwar - in der Reihenfolge ihrer Häufigkeit - $\mathrm{Pt}, \mathrm{Ag}$, $\mathrm{Cu}, \mathrm{Mg}$ sowie Spuren von $\mathrm{Au}, \mathrm{Fe}$ und $\mathrm{Ca}$.

Die Zahl der Schaltungen wurde jeweils nach der Kondensatorenergie so gewählt, daß das $\mathrm{zu}$ messende Metallvolumen zwischen $10^{-6}$ und $3 \cdot 10^{-5} \mathrm{~cm}^{3}$ lag. In diesem Intervall waren die Ablagerungen meist symmetrisch aufgebaut und ermöglichten eine optische Volumenbestimmung mit einer Genauigkeit von $\pm 10 \%$.

Das Ergebnis der Messungen zeigt Abb. 1. Bezieht man das übergeführte Metallvolumen auf die Einheit der Kondensatorenergie, so ist die Stoffwanderung über einem den Elektrodenwerkstoff kennzeichnenden Schwellenwert $V_{\min }$ von der Spannung unabhängig. Der Anodenabbau kann in diesem Spannungsbereich dem Anodenbogen zugeordnet werden ${ }^{7}$.

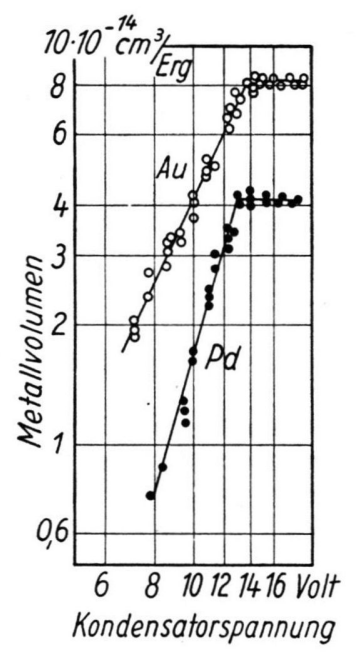

Abb. 1.

Unterhalb dieses Grenzwertes wird die Stoffwanderung zum größten Teil durch einen anderen Vorgang hervorgerufen. Reicht bei der ersten Berührung der Kontakte die Stromstärke zu einer Verdampfung der metallischen Brücken aus, so hebt die Selbstinduktion des Entladekreises die Spannung auf $V_{\min }$ an, und ein Ausschaltbogen zündet trotz der niederen Kondensatorspannung.

Die Höhe der beiden Kurven und ihre Neigung im ersten Abschnitt sind in geringem Maße von der Kondensatorenergie abhängig. $\mathrm{Da}$ jedoch nachstehend nur aus der Lage der Knickstellen einige Folgerungen abzuleiten sind, soll an dieser Stelle nicht näher darauf eingegangen werden.

7 L. H. Germer u. F. E. Haworth, J. Appl. Phys. 20, 1085 [1949].

8 H. B. Michaelson, J. Appl. Phys. 21, 536 [1950].

9 B. GäNGER, Der elektrische Durchschlag von Gasen, Springer 1953, S. 32 .
Die Grenzspannung $V_{\min }$ beträgt für Feingold-Kontakte $13,5 \pm 0,3$ Volt, für Palladium-Kontakte nur $12,8 \pm 0,2$ Volt. Werte in dieser Höhe sind nach der Hypothese einer Zündung des kurzen Bogens durch Feldemission $\mathrm{zu}$ erwarten ${ }^{4}$, denn sie entsprechen etwa der Summe von Elektronen-Austrittspotential $\varphi$ und Ionisierungsspannung $V_{\mathrm{I}}$ des zugehörigen Metalldampfes (Tab. 1). Die Differenz zwischen $V_{\min }$ und den berechneten Zündspannungen ist für beide Metalle gleich $-0,3$ Volt. Da sie jedoch bei Au noch an der Grenze des Meßfehlers liegt und die Werte der Austrittsarbeiten mit einer Unsicherheit der gleichen Größe behaftet sind $^{8}$, kann vorerst noch nicht auf eine wirkliche Diskrepanz geschlossen werden.

In Tab. 1 ist außerdem noch die Brennspannung des Anodenbogens zwischen Pd-Elektroden angegeben ${ }^{3}$,

\begin{tabular}{|l|c|c|}
\hline & $\mathrm{Au}$ & $\mathrm{Pd}$ \\
\hline $\begin{array}{l}\text { Zündspannung } V_{\min } \\
\text { Brennspannung } \\
\text { GERMER-BoYLE }^{3}\end{array}$ & $13,5 \pm 0,3$ & $12,8 \pm 0,2$ Volt \\
$\varphi^{8}+V_{\mathrm{I}}{ }^{9}$ & - & $9-12$ \\
\hline
\end{tabular}

Tab. 1.

welche wesentlich tiefer als die Zündspannung liegt. Aus den Untersuchungen von Germer und Smith mit einem hochauflösenden Oszillographen ${ }^{10}$ ist andererseits bekannt, daß sich die Brennspannung nur wenig mit der Bogendauer ändert. Daraus folgt, daß die Spannung innerhalb $10^{-9} \mathrm{sec}$ nach Beginn der Entladung, d. h. etwa dem Hundertfachen der Ionenlaufzeit, von $V_{\text {min }}$ auf die konstante Brennspannung absinkt.

Die eindeutige Abhängigkeit der Zündspannung von den physikalischen Daten des Elektrodenmetalls und der monotone Verlauf der Meßkurven nach höheren Spannungen hin zeigen auch, daß die Atmosphäre und adsorbierte Gasschichten bei diesen niederen Spannungen nur einen sehr geringen Einfluß auf die Ausbildung der Entladung haben können. Die Zeit zwischen zwei aufeinanderfolgende Schaltungen ist mindestens $10^{6}$-mal so lang wie die Bedeckungszeit reiner Edelmetallflächen mit einer einatomigen Schicht unter Normalbedingungen ${ }^{11}$, so daß auch durch Metallablagerungen neugebildete Oberflächen mindestens mit einer Molekülschicht bedeckt sind. Wären leicht ablösbare Gasmoleküle für die Erzeugung der ersten Ionen notwendig, wie verschiedentlich angenommen wurde, so müßten in beiden Meßkurven Abweichungen von der Geraden bei den Schwellenwerten, die der Stickstoff- oder SauerstoffIonisierung entsprechen, im Sinne einer gesteigerten Verdampfungsrate auftreten. Das Fehlen dieser Abweichungen bestärkt die Annahme, daß der Durchschlagsmechanismus in Luft bei niederen Spannungen mit dem des kurzen Hochvakuumbogens ${ }^{12}$ identisch ist.

10 L. H. Germer u. J. L. Smith, J. Appl. Phys. 23, 553 [1952]. 11 nach einer privaten Mitteilung von H. D. Hagstrum.

12 W. S. Boyle, P. Kisliuk u. L. H. Germer, J. Appl. Phys. 26, 720 [1955]. 